\title{
MicroRNA-182 Promotes Tumor Cell Growth by Targeting Transcription Elongation Factor A-like 7 in Endometrial Carcinoma
}

\author{
Ying Guo Ying Liao $^{\mathrm{a}}$ Chunyan Jia ${ }^{\mathrm{b}}$ Jianlin Ren ${ }^{\mathrm{c}}$ Jianchao Wang ${ }^{\mathrm{a}}$ Ting Lia \\ aDepartment of Gynaecology, Shanghai Traditional Chinese Medicine Hospital, Shanghai, ${ }^{b}$ Department \\ of Ultrasonograpy, Shanghai Traditional Chinese Medicine Hospital, Shanghai, 'Department of \\ Oncology, Shanghai Traditional Chinese Medicine Hospital, Shanghai
}

\section{Key Words}

miR-182 • Endometrial carcinoma • TCEAL7

\begin{abstract}
Background/Aims: Endometrial carcinoma (EC) is the most common gynecological malignancy among women worldwide. Despite its prevalence, the molecular mechanisms underlying endometrial carcinogenesis are poorly understood. The purpose of this study was to examine the role of microRNA-182 and its target gene transcription elongation factor A-like 7 (TCEAL7) in EC. Methods: The expression of miR-182 in human normal endometrial epithelial cells (NEEC) and in three human endometrial carcinoma cell lines (HEC-1B, RL95-2 and AN3CA) was measured by qRT-PCR, and the MRNA and protein expression of TCEAL7 were assessed in the same three endometrial carcinoma cell lines and NEEC by qRT-PCR and western blotting, respectively. Subsequently, the target of miR-182 was predicted by bioinformatics and confirmed using a luciferase assay. Cell proliferation and colony formation of RL95-2 cells were examined by MTT assay and crystal violet staining, respectively. The expression of NFkB-p65, c-Myc and cyclin D1 proteins was determined by Western blot analysis. Results: MiR-182 was significantly upregulated and TCEAL7 was downregulated in EC cell lines compared to NEEC. We showed that MiR-182 binds directly to a conserved 8 bp sequence in the 3'-UTR of TCEAL7, and inhibition of miR-182 upregulated TCEAL7 mRNA and protein expression to levels comparable to those induced by lentiviral-mediated overexpression of TCEAL7. MiR182 inhibition decreased cell proliferation and colony formation ability, downregulated the expression of the pro-proliferative genes c-Myc and cyclin D1, and inhibited NFKB activation, and these effects were mimicked by TCEAL7 overexpression. Conclusions: miR-182 acts as an oncogenic miRNA in EC, promoting cell proliferation by targeting the tumor suppressor gene TCEAL7 and modulating the activity of its downstream effectors c-Myc, cyclin D1 and NFKB.
\end{abstract}

Copyright (C 2013 S. Karger AG, Basel

Ying Liao, MD, Ph.D 
Guo et al.: MiR-182 Promotes Tumor Cell Growth by Targeting TCEAL7 in EC

\section{Introduction}

Endometrial carcinoma (EC) is the fourth most common cancer among women in the United States [1] and the seventh most common cancer among women worldwide [2]. Although several prognostic factors have been identified and different treatment strategies have been developed for this disease, to date, no effective therapy has been established and the pathogenesis of endometrial cancer is not yet fully understood [3]. Therefore, the identification of EC-specific biomarkers for the early detection of this disease and a better understanding of the molecular mechanisms underlying EC is essential for the development of effective treatment strategies.

The identification of microRNAs (miRNAs) and their role in the posttranscriptional regulation of gene expression has led to new hypotheses on the pathogenesis of endometrial cancer [4-6]. MiRNAs are small endogenous non-coding RNAs (19-25 nucleotides in length) that negatively regulate gene expression through incomplete base-pairing to the 3 ' untranslated region (3'-UTR) of target mRNAs [7]. Deregulation of miRNAs is associated with the pathogenesis of several diseases including cancer, and tumor-associated miRNAs can function as tumor suppressors or oncogenes depending on whether they target oncogenes or tumor suppressor genes $[8,9]$. Studies have identified miRNAs upregulated or downregulated in EC, among which miR-182 was shown to be significantly upregulated in endometrial carcinoma tissues compared with complex atypical hyperplasia, simple hyperplasia and normal endometrial tissues [10-12].

The dysregulation of miR-182 in endometrioid endometrial carcinoma tissues was confirmed by global expression profiling in a study in which TCEAL7 was identified as the predicted target gene of miR-182 and shown to be downregulated in EC [13]. TCEAL7 was first cloned as a proapoptotic nuclear protein and shown to function as a tumor suppressor gene downregulated in ovarian cancer [14]. TCEAL7 is also downregulated in gastric cancer, and low TCEAL7 expression is associated with large tumors, high histological grade, poor nodal status and poor prognosis of gastric adenocarcinoma patients [15]. Furthermore, the downregulation of TCEAL7 in breast, brain and prostate cancers indicates that it may play an important role in carcinogenesis, possibly through its effect on the NFKB pathway and the pro-proliferative genes cyclin D1 and c-Myc [16-18].

In the present study, we show that miR-182 is upregulated and its target gene TCEAL7 is downregulated in EC cell lines. We identified the miR-182 target site in the 3'-UTR of TCEAL7 and showed that miR-182 interacts directly with and regulates the expression of TCEAL7, modulating cell proliferation and colony formation ability through the TCEAL7 mediated control of c-Myc and cyclin D1 expression and NFKB signaling.

\section{Materials and Methods}

\section{Ethics statement}

Human endometrial samples were collected after obtaining written informed consent from all patients. The study was approved by the Institutional Review Board of Shanghai Traditional Chinese Medicine Hospital.

\section{Cell lines and culture}

The human endometrial carcinoma cell lines used were HEC-1B, derived from moderately differentiated endometrial adenocarcinoma; RL95-2, derived from moderately differentiated adenosquamous carcinoma of the endometrium; and AN3CA, derived from undifferentiated endometrial adenocarcinoma. All the three cell lines were purchased from the American Tissue Culture Collection and cultured in Eagle's Minimum Essential Medium (Invitrogen, Carlsbad, CA, USA) supplemented with 10\% fetal bovine serum (HyClone, Logan, UT, USA) at $37^{\circ} \mathrm{C}$ with $5 \% \mathrm{CO}_{2}$ and $95 \%$ humidified air. Normal human endometrial epithelial cells (NEEC) were generated from human endometrium samples obtained from biopsies of women (22-23 years of age) who underwent surgery for minor gynecological issues and had no underlying endometrial pathology. 
Guo et al.: MiR-182 Promotes Tumor Cell Growth by Targeting TCEAL7 in EC

None of them had received hormonal therapy in the 3 months preceding sample collection. Endometrial samples were minced into fragments $<1 \mathrm{~mm}$ and subjected to mild collagenase digestion. Endometrial epithelial cells were isolated as described previously [19] and cultured to confluency in a steroid-depleted medium composed of 75\% Dulbecco's Modified Eagle Medium and 25\% MCDB-105 (Sigma, St. Louis, MO) supplemented with antibiotics, $10 \%$ human albumin and $5 \mathrm{mg} / \mathrm{mL}$ insulin (Sigma).

\section{Quantitative real-time PCR}

Total RNA was extracted from EC cells using the Trizol reagent (Invitrogen) according to the manufacturer's instructions. For microRNA analysis, quantitative real-time PCR (qRT-PCR) was performed using the TaqMan MicroRNA Reverse Transcription Kit, TaqMan Universal PCR Master Mix (Applied Biosystems, Foster City, CA, USA), and the corresponding primers. U6 small nuclear (sn) was used as an internal control to normalize miR-182 levels. For mRNA analysis, qRT-PCR was performed using the TaqMan High-Capacity cDNA Reverse Transcription Kit, TaqMan Fast PCR Master Mix (Applied Biosystems) and the corresponding primers. GAPDH was used as an internal control to normalize TCEAL7. qRT-PCR reactions were performed in triplicate on a RealPlex4 real-time PCR detection system from Eppendorf Co. LTD (Germany). The following primers for qRT-PCR were used: miR-182: forward, 5'-GAG AAC AGC AGG TCC AGC AT-3'; reverse: 5'-CTT CCT CAG CAC AGA CCG AG-3'. U6: forward, 5'-CTC GCT TCG GCA GCA CA-3'; reverse, 5'-AAC GCT TCA CGA ATT TGC GT-3'. TCEAL7: forward, 5'-ACA TCA TGC AAA AAC CCT GCA A-3'; reverse, 5'ACG GTC CCG AGA ATG CCT AT-3'. GAPDH: forward, 5'-CCC ATG TTC GTC ATG GGT GT-3'; reverse, 5'-CCG TTC AGC TCA GGG ATG AC-3'. PCR parameters were as follows: $10 \mathrm{~min}$ at $95^{\circ} \mathrm{C}$, and then 40 amplification cycles of $15 \mathrm{~s}$ at $95^{\circ} \mathrm{C}, 1 \mathrm{~min}$ at $60^{\circ} \mathrm{C}$. The threshold cycle (Ct) was defined as the fractional cycle number at which the fluorescence passes the fixed threshold and the relative miRNA or mRNA expression was calculated using the $2^{-\Delta \Delta C t}$ method [20].

\section{Western blot analysis}

Total protein lysates were resolved by 10\% SDS-PAGE and transferred to polyvinyl difluoride membranes (Millipore, Bedford, MA). After blocking in Tris buffered saline containing 0.1\% Tween-20 (TBS-T) with 5\% nonfat dry milk for 30 min, membranes were washed 4 times in TBS-T and incubated with primary antibodies overnight at $4^{\circ} \mathrm{C}$. Primary antibodies were all obtained from Abcam (Cambridge, MA) and used at the following dilutions: anti-TCEAL7 (1/300), anti-NFKB-p65 (1/500), anti-c-Myc (1/500), anti-cyclin D1 (1/500), anti- $\beta$ actin (1/1000). After extensive washing, membranes were incubated with horseradish peroxidase-linked goat polyclonal anti rabbit IgG secondary antibodies at a dilution of 1:2000 for $1 \mathrm{~h}$ at room temperature. Immunoreactivity was detected by enhanced chemiluminescence (ECL kit, Pierce Biotechnology) and exposure to radiography film. $\beta$-actin served as the loading control.

\section{Luciferase reporter assay}

The 3'-UTR of TCEAL7 was amplified with the following primers: forward, 5'-CCA TAG GCA TTC TCG GGA CC-3'; reverse, 5'-TCT GGC TAA GAG ATC CAG AGG T-3'. DNA fragments were cloned into the unique Xbal site downstream of the luciferase gene in the pRL-TK vector to generate the plasmid pRL-TK-TCEAL7-3' UTR. To generate the mutant reporter vector, the nucleotides TTGCCAAA located at residues 410-417 of the TCEAL7-3'-UTR corresponding to the hsa-mir-182 seed sequence were mutated to ATCCGATA. Reporter constructs were co-transfected into HEC-1B (or RL95-2 or AN3CA) cells in 24-well plates together with the Firefly plasmid pGL3 (Promega, Madison, WI) and miR-182 mimics (purchased from Ambion; double strand RNA, sense: 5'-UUU GGC AAU GGU AGA ACU CAC ACU-3', antisense: 5'-AGU GUG AGU UCU ACC AUU GCC AAA-3') or negative control mimics miRNA (Ambion, Austin, TX; double strand RNA, sense: 5'-UUC UCC GAA CGU GUC ACG UUU-3', antisense: 5'-AAA CGU GAC ACG UUC GGA GAA-3'). Cells were harvested at $48 \mathrm{~h}$ post-transfection by treatment with trypsin, lysed in $1 \times$ Passive Lysis Buffer (Promega), and centrifuged at $12,000 \mathrm{rpm}$ for $5 \mathrm{~min}$. The supernatant was assayed for Firefly and Renilla luciferase activities following the Dual Luciferase Reporter Assay System (Promega) protocol.

Inhibition of miR-182 or overexpression of TCEAL7 in RL95-2 cells

A miR-182 inhibitor (Ambion, double strand RNA, sense: 5'-UAA GCC GCG AGC ACU CUG CCU GCA C-3', antisense: 5'-GUG CAG GCA GAG UGC UCG CGG CUU A-3') or a negative control inhibitor miRNA (Ambion, double strand RNA, sense: 5'-UCA GAU GCA CAG ACU CCC GUG GU-3', antisense: 5'- 
Fig. 1. MiR-182 is upregulated and TCEAL7 is downregulated in endometrial carcinoma cell lines. (A) The expression of miR-182 in human normal endometrial epithelial cells (NEEC) and in three human endometrial carcinoma cell lines (HEC-1B, RL95-2 and AN3CA) was measured by qRT-PCR with snRNA U6 level as an internal control. (B) The mRNA expression of TCEAL7 was assessed in the same three endometrial carcinoma cell lines and NEEC with GAPDH mRNA level as the control, and (C) TCEAL7 protein levels were analyzed by western blotting with $\beta$-actin as the loading control. (D) Quantitative analysis of TCEAL7 protein levels normalized to $\beta$-actin levels. All values are the means \pm SD of three replicates, ${ }^{* *} p<0.01$ vs NEEC.

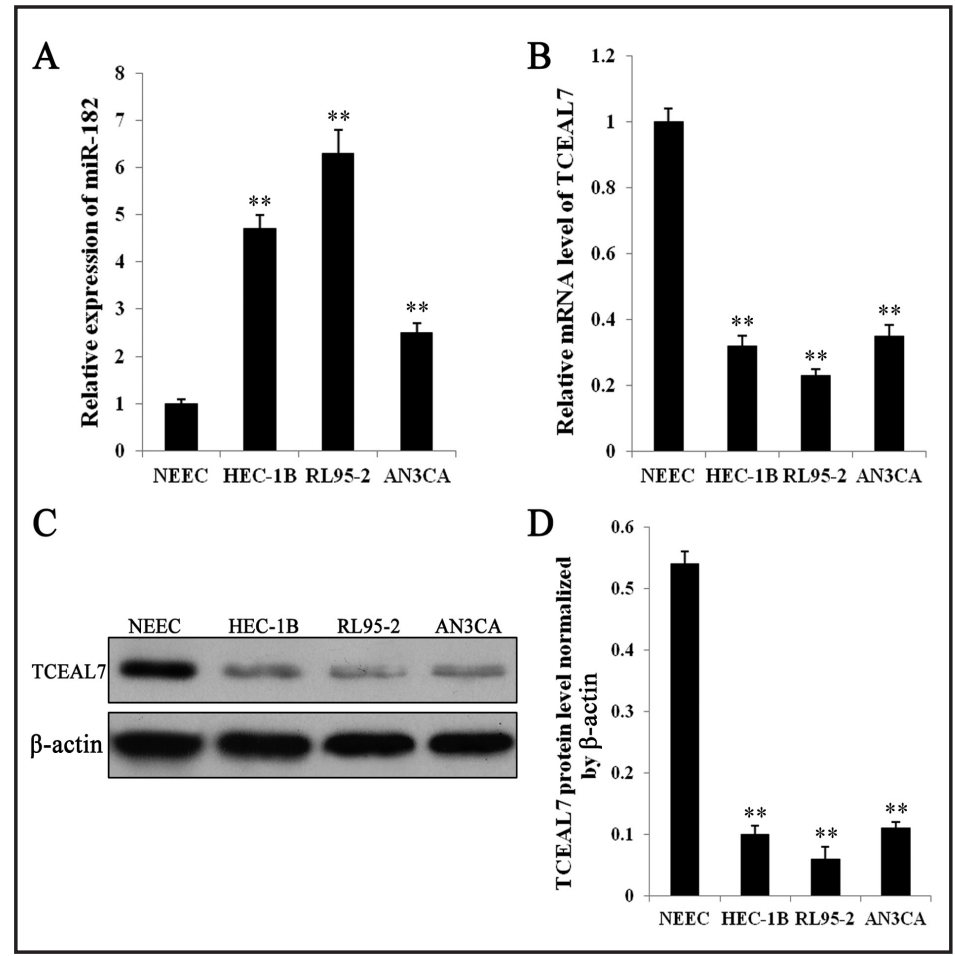

ACC ACG GGA GUC UGU GCA UCU GA-3') were transfected into RL95-2 cells using Lipofectamine 2000 (Invitrogen) following the manufacturer's instructions. For overexpression of TCEAL7, the full length TCEAL7 cDNA (gi|299758466|ref|NM_152278.3| Homo sapiens transcription elongation factor A (SII)like 7 (TCEAL7) was amplified using the following primers (restriction enzyme cutting sites, EcoRI and NotI: forward, TCEAL7- EcoRI: AGGAATTCAGCTGACGTGAACCGAAGTC; reverse, TCEAL7-NotI: TGCGGCCGCTCCAAAGGCGATTGACAAGT). The full-length TCEAL7 cDNA fragment was cloned into the pCDH-CMV vector (System Biosciences, SBI, Mountain View, CA). Recombinant lentivirus was produced by transient transfection in 293T cells using Lipofectamine 2000 (Invitrogen). Medium containing infectious lentiviruses was harvested at $48 \mathrm{~h}$ post-transfection and filtered through $0.45-\mu \mathrm{m}$-pore cellulose acetate filters. RL95-2 cells were incubated with infectious lentivirus medium. Empty lentiviral vector was used as the negative control.

\section{Cell proliferation (MTT assay)}

RL95-2 cells transfected with miR-182 inhibitor or a negative control inhibitor miRNA or expressing TCEAL7 lentivirus or pCDH-CMV vector lentivirus were seeded at a density of 5000 cells per $200 \mu \mathrm{L}$ medium in each well of a 96 well plate. At $0,1,2,3,4$, and 5 days after transfection, the medium was removed by suction and replaced by serum free medium containing $1 \mathrm{mg} / \mathrm{mL} 3$-(4,5-dimethylthiazol-2-yl)-2,5diphenyltetrazolium bromide (MTT) and incubated at $37^{\circ} \mathrm{C}$ for $4 \mathrm{~h}$. After removing the MTT solution, the formazan precipitate was dissolved with $100 \mu \mathrm{L}$ DMSO and absorbance was read at 570 and $600 \mathrm{~nm}$ in a microplate reader (BioTek, Bad Friedrichshall, Germany). Untransfected cells were used as the normal controls.

\section{Colony formation assay}

RL95-2 cells transfected with miR-182 inhibitor or a negative control inhibitor miRNA or expressing TCEAL7 lentivirus or pCDH-CMV vector lentivirus were seeded in 6-well culture plates at a density of 500 cells per well to form colonies. After 7 days, colonies were fixed in $4 \%$ paraformaldehyde and stained in a $0.1 \%$ crystal violet solution. Untransfected cells were used as the normal controls.

\section{Statistical analysis}

Data were expressed as the mean \pm SE from at least three independent experiments. Differences were analyzed using the Student's t-test. $\mathrm{P}<0.05$ or $\mathrm{P}<0.01$ was considered statistically significant. 


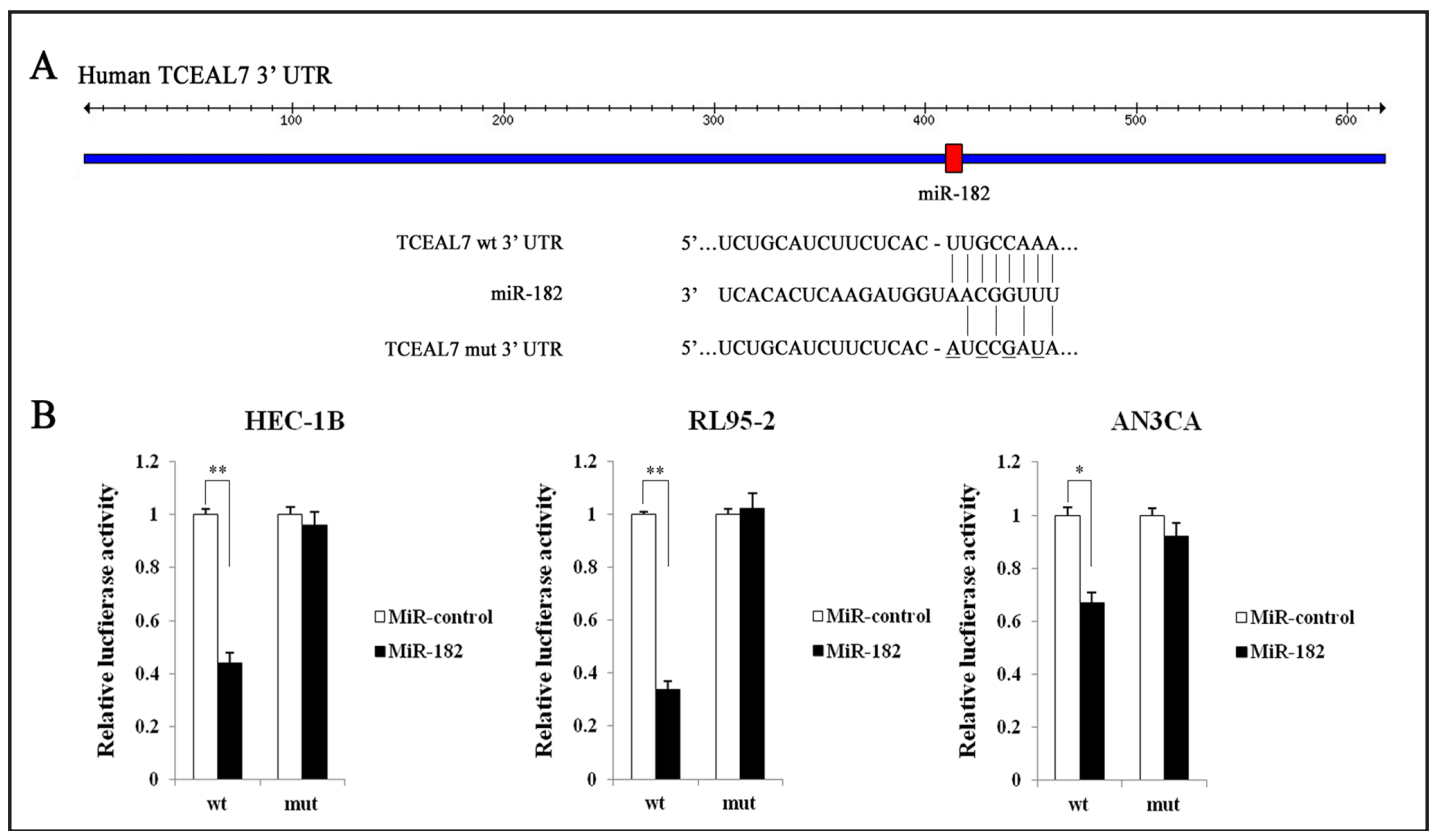

Fig. 2. TCEAL7 is a target gene of miR-182. (A) Sequence alignment of miR-182 and the 3'-UTR of TCEAL7 containing the conserved 8-bp "seed" sequence predicted as the miR-182 binding site. The paired miR-182 seed sequence and the seed-recognizing site in the wild-type (wt) and mutant (mut) 3'-UTR of TCEAL7 are indicated. (B) Luciferase assay of HEC-1B, RL95-2 and AN3CA cells co-transfected with fluorescent reporter vectors containing either wt or mut binding sites and miR-182 mimics (MiR-182) or negative miRNA control mimics (MiR-control). Luciferase activities were measured and normalized to the controls, and expressed as relative luciferase activity. All values are the means \pm SD of three replicates. ${ }^{*} \mathrm{p}<0.05,{ }^{* *} \mathrm{p}<0.01$.

\section{Results}

miR-182 is upregulated and TCEAL7 is downregulated in endometrial carcinoma

The expression of miR-182 and its target TCEAL7 was examined in three human endometrial carcinoma cell lines (HEC-1B, RL95-2 and AN3CA) and in normal endometrial epithelial cells (NEEC) by qRT-PCR and western blotting. The results showed that miR182 mRNA expression was significantly upregulated whereas TCEAL7 mRNA and protein expressions were significantly downregulated in carcinoma cells compared to normal epithelial cells $(\mathrm{P}<0.01$ all) (Fig. 1A, B and C). Quantification of bands by densitometry showed that TCEAL7 protein levels were reduced by approximately 5-fold in HEC-1B and AN3CA and 10-fold in RL95-2 cells compared to NEEC levels (Fig. 1D).

TCEAL7 is a target gene of miR-182

To explore the association between miR-182 and its target gene TCEAL7, the TargetScan algorithm was used to predict the target sites for miR-182 in the 3'-UTR of TCEAL7 (Fig. 2A). To confirm that TCEAL7 is a direct target of miR-182, TCEAL7 wild-type (WT) or a mutant (Mut) TCEAL7 3'-UTR generated by mutation of residues 401-417 of the seed sequence was subcloned into a luciferase reporter vector and co-transfected with miR-182 mimics or negative control mimics (miR-control) into HEC-1B, RL95-2 and AN3CA cells. Luciferase reporter assays showed that miR-182 significantly decreased the luciferase activity of the WT 3'-UTR but not that of the mutant 3'-UTR of TCEAL7 in HEC-1B, RL95-2 (P<0.01) and AN3CA $(\mathrm{P}<0.05)$ cells (Fig. $2 \mathrm{~B})$, confirming that TCEAL7 is a target gene of miR-182 in EC. 
Fig. 3. MiR-182 negatively regulates the expression of TCEAL7. (A) RL95-2 cells were transfected with a specific miRNA inhibitor (miR-182 inhibitor) or a negative control inhibitor miRNA (Negative control) for $48 \mathrm{~h}$ and the levels of miR-182 were measured by qRT-PCR with snRNA U6 level as an internal control. (B) RL95-2 cells were co-transfected with miR-182 inhibitor or negative control and lentivirus expressing TCEAL7 (lenti-TCEAL7) or the pCDH-CMV vector control lentivirus (lenti-vector) for $48 \mathrm{~h}$. TCEAL7 mRNA expression was assessed by qRT-PCR and normalized to the levels of GAPDH, and (C) TCEAL7 protein levels were assessed by western blotting with $\beta$-actin as the loading control. (D) Quantitative analysis of TCEAL7 protein levels normalized to the levels of $\beta$-actin. All values represent the means \pm SD of three replicates. ${ }^{* *} \mathrm{p}<0.01$ vs. untreated (not transfected) RL95-2 cells.

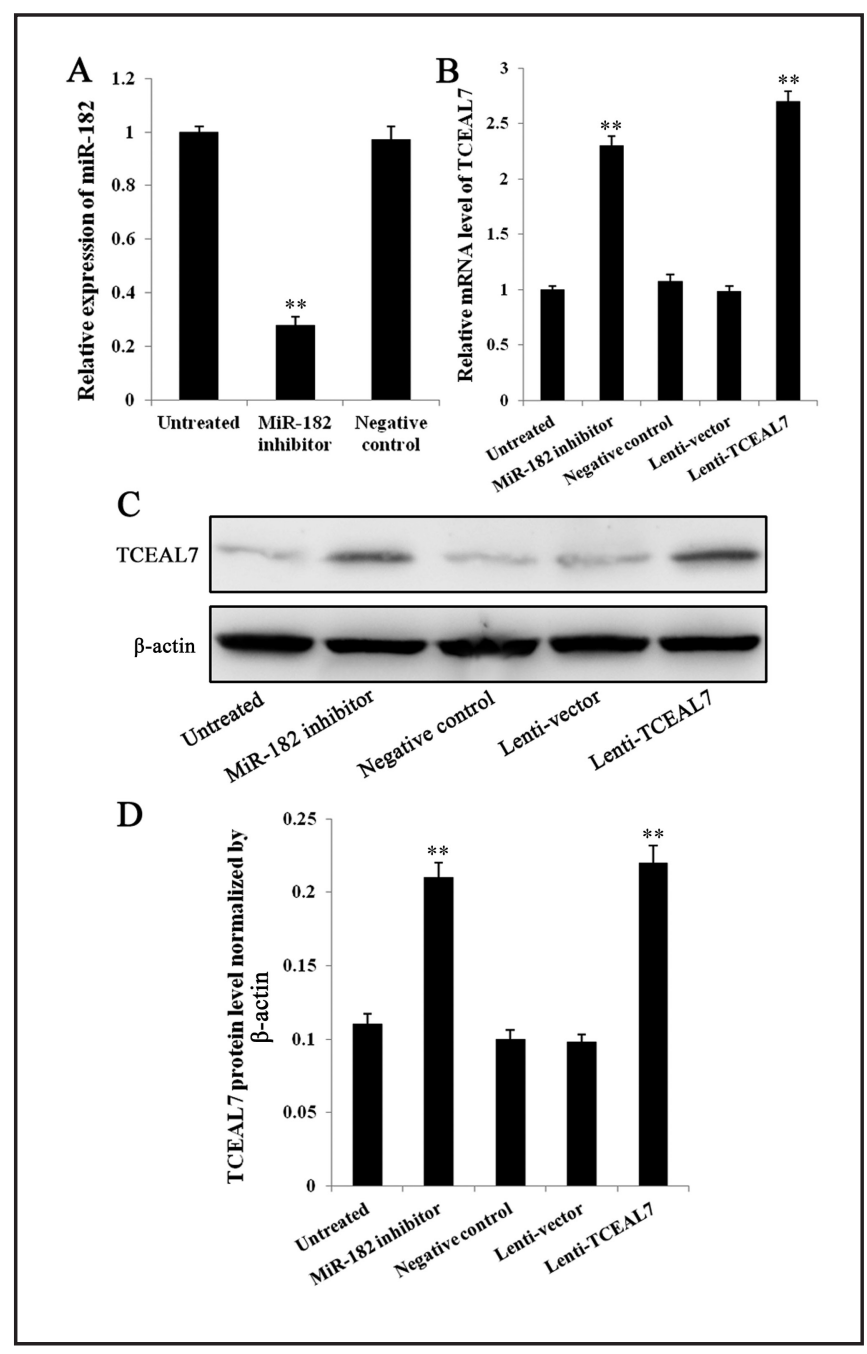

miR-182 negatively regulates TCEAL7 expression

To further examine whether miR-182 exerts its function by modulating the expresssion of TCEAL7 in EC, RL95-2 cells were transfected with a miR-182 inhibitor, which effectively decreased miR-182 levels (Fig. 3A). Inhibition of miR-182 significantly upregulated TCEAL7 expression to a level comparable to that induced by lentiviral vector-mediated overexpression of TCEAL7 (Fig. 3B). Western blot analysis showed that inhibition of miR182 resulted in an approximately two-fold increase in TCEAL7 protein levels compared to untreated or negative control transfected cells, which was similar to the effect of lentiviralmediated TCEAL7 overexpression and confirmed the results of qRT-PCR (Fig. 3C and D).

miR-182 promotes cell proliferation and colony formation via TCEAL 7 regulation of NFKB activity and c-Myc and cyclin D1 expression

The function of miR-182 in EC was further examined by assessing the effect of miR-182 inhibition and TCEAL7 overexpression on cell proliferation and colony formation in RL95-2 cells. Inhibition of miR-182 significantly suppressed cell proliferation (Fig. 4A) and colony formation ability (Fig. 4B) ( $\mathrm{P}<0.01$, all) to levels comparable to those induced by TCEAL7 overexpression. To examine the mechanism of inhibition of cell proliferation by miR-182 modulation of TCEAL7 activity, we assessed NFKB activation, and c-Myc and cyclin D1 levels in cells transfected with a miR-182 inhibitor or subjected to lentiviral-mediated TCEAL7 overexpression. The results showed that miR-182 inhibition significantly downregulated the expression of the NFKB p65 subunit, c-Myc and cyclin D1 compared to untreated and 


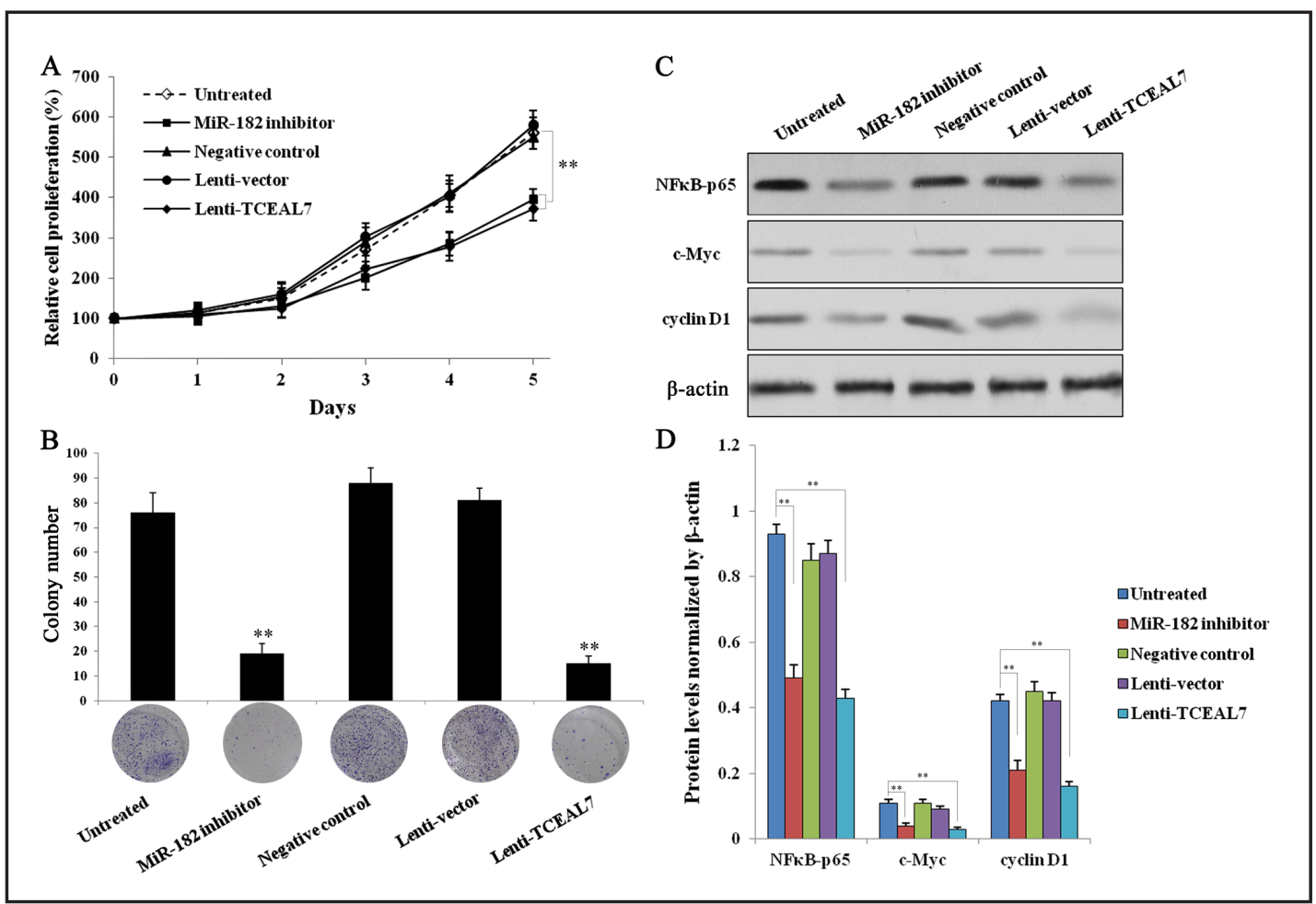

Fig. 4. MiR-182 regulates tumor cell proliferation, c-Myc and cyclin D1 expression and NFkB activity by targeting TCEAL7 in endometrial cancer. (A) RL95-2 cells were co-transfected with miR-182 inhibitor or negative control and lentivirus expressing TCEAL7 (lenti-TCEAL7) or the pCDH-CMV vector control lentivirus (lenti-vector), and cell proliferation was assessed by MTT assay. Untransfected cells were used as control. (B) colony formation assay. (C) Western blot analysis of NFKB-p65, c-Myc and cyclin D1 levels with $\beta$-actin as the loading control. (D) Quantitative analysis of NFKB-p65, c-Myc and cyclin D1 protein levels normalized to the levels of $\beta$-actin. All values represent the means \pm SD of three replicates. ${ }^{* *} p<0.01$ vs. untreated (not transfected) RL95-2 cells.

negative-control transfected cells, and this effect was mimicked by TCEAL7 overexpression (Fig. 4C and D). Taken together, these results indicate that the effect of miR-182 is mediated by the transcriptional modulation of genes involved in proliferation and cell cycle progression by its target gene TCEAL7.

\section{Discussion}

In the present study, we showed that miR-182 is upregulated and its target gene TCEAL7 is downregulated in EC cell lines, and inhibition of miR-182 increases TCEAL7 expression levels and suppresses cell proliferation and colony formation, suggesting that miR-182 acts as an oncogene in EC by targeting the tumor-suppressor TCEAL7. Several miRNAs have been identified that are up- or down-regulated in EC. Among miRNAs upregulated in EC, miRNAs $(182,183,200 \mathrm{a}, 34 \mathrm{a}$ and 95) are strongly associated with tumor progression and lymph node metastases [13, 21]. MiR-182 upregulation was also reported in ovarian cancer [22, 23] and cervical cancer [24,25], suggesting the possibility of a common underlying mechanism of carcinogenesis in these gynecological malignancies. Considering that endometrial cancer is an estrogen-dependent malignancy and that miRNAs were shown to be regulated by estradiol [26], the association between miRNAs and the estrogen response in EC merits further investigation. 
MiR-182 functions as oncogene or tumor-suppressor in different human malignancies. The tumor suppressor function of miR-182 was shown in uveal melanoma, where it regulates cell proliferation, migration and invasion in a p53 dependent manner by targeting MITF, BCL2 and cyclin D2 [27]. In lung cancer, miR-182 suppresses lung tumorigenesis by targeting RGS17 [28], and in gastric cancer, miR-182 binds directly to the oncogene cAMP-responsive element-binding protein 1, suppressing tumor cell growth [29]. MiR-182 is overexpressed and functions as an oncogene in several human cancers such as breast cancer, where it targets FOXO1 together with miR-27a and miR-96 [30]; in melanoma, it promotes anchorage independent growth, invasion and metastasis [31]; and in ovarian carcinoma, miR-182 promotes cell proliferation, invasion and chemoresistance by targeting programmed cell death 4 [32]. MiR-182 overexpression in colorectal cancer is associated with adverse clinical characteristics and poor prognosis [33], and in hepatocellular carcinoma, it is correlated with metastatic behavior [34]. MiR-182 is also overexpressed in high grade serous ovarian carcinoma [35], and prostate carcinoma [36]. In the present study, miR-182 was overexpressed in three different EC cell lines and it negatively regulated the expression of its target TCEAL7, a tumor suppressor gene.

We showed that miR-182 binds directly to the 3'- UTR of TCEAL7, modulating its expression and the TCEAL7-mediated regulation of essential downstream molecules involved in cell proliferation and cell cycle progression. TCEAL7 interacts with E-box sequences of the Myc target gene cyclin D1, regulating Myc activity and cyclin D1 expression and thus cell proliferation and malignant transformation [16]. We showed that overexpression of TCEAL7 decreased cell proliferation and colony formation ability, and downregulated the expression of c-Myc and cyclin D1, suggesting that the loss of TCEAL7 in EC may specifically favor the survival of cancer cells. Furthermore, exogenous expression of TCEAL7 significantly decreased the levels of the NFKB p65 subunit, which is in agreement with prior results showing that TCEAL7 negatively regulates $\mathrm{NF} \kappa \mathrm{B}$ transcriptional activity in ovarian cancer [18]. These effects of TCEAL7 were mimicked by miR-182 inhibition, and miR-182 is significantly upregulated in EC. Taken together, these results suggest that the oncogenic effects of miR-182 may be mediated at least in part by the downregulation of TCEAL7 in EC, leading to the activation of $\mathrm{c}-\mathrm{Myc}$ and $\mathrm{NF \kappa B}$ and the upregulation of cyclin D1 expression.

In conclusion, the present study showed that miR-182 is upregulated and its target gene TCEAL7 is downregulated in EC cell lines. Inhibition of miR-182 upregulated TCEAL7 expression, resulting in the inhibition of cell proliferation and colony formation, the downregulation of c-Myc and cyclin D1 expression, and the suppression of NFKB signaling. Our results indicate that miR-182 functions as an oncogenic miRNA in EC by targeting the tumor suppressor gene TCEAL7, and suggest miR-182 as a potential therapeutic target in EC.

\section{Acknowledgements}

This study was supported by grants from the Science and Technology Commission of Shanghai Municipality (No. 12401902901 and No. 10DZ1974600).

\section{References}

Jemal A, Siegel R, Xu J, Ward E: Cancer statistics, 2010. CA Cancer J Clin 2010;60:277-300.

Dizon DS: Treatment options for advanced endometrial carcinoma. Gynecol Oncol 2010;117:373-381.

Dong P, Kaneuchi M, Konno Y, Watari H, Sudo S, Sakuragi N: Emerging therapeutic biomarkers in endometrial cancer. Biomed Res Int 2013;2013:130362.

-4 Skaftnesmo KO, Prestegarden L, Micklem DR, Lorens JB: Micrornas in tumorigenesis. Curr Pharm Biotechnol 2007;8:320-325. 
Guo et al.: MiR-182 Promotes Tumor Cell Growth by Targeting TCEAL7 in EC

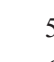

$\rightarrow 6$

$>7$

$>8$

$>9$

${ }_{10}$

Temkin SM, Fleming G: Current treatment of metastatic endometrial cancer. Cancer Control 2009;16:38-45. Valencia-Sanchez MA, Liu J, Hannon GJ, Parker R: Control of translation and mrna degradation by mirnas and sirnas. Genes Dev 2006;20:515-524.

Rana TM: Illuminating the silence: Understanding the structure and function of small rnas. Nat Rev Mol Cell Biol 2007;8:23-36.

Esquela-Kerscher A, Slack FJ: Oncomirs - micrornas with a role in cancer. Nat Rev Cancer 2006;6:259-269. Wang D, Qiu C, Zhang H, Wang J, Cui Q Yin Y: Human microrna oncogenes and tumor suppressors show significantly different biological patterns: From functions to targets. PLoS One 2010;5.

Lee H, Choi HJ, Kang CS, Lee HJ, Lee WS, Park CS: Expression of mirnas and pten in endometrial specimens ranging from histologically normal to hyperplasia and endometrial adenocarcinoma. Mod Pathol 2012;25:1508-1515.

Ratner ES, Tuck D, Richter C, Nallur S, Patel RM, Schultz V, Hui P, Schwartz PE, Rutherford TJ, Weidhaas JB: Microrna signatures differentiate uterine cancer tumor subtypes. Gynecol Oncol 2010;118:251-257.

Wu W, Lin Z, Zhuang Z, Liang X: Expression profile of mammalian micrornas in endometrioid adenocarcinoma. Eur J Cancer Prev 2009;18:50-55.

Chung TK, Cheung TH, Huen NY, Wong KW, Lo KW, Yim SF, Siu NS, Wong YM, Tsang PT, Pang MW, Yu MY, To KF, Mok SC, Wang VW, Li C, Cheung AY, Doran G, Birrer MJ, Smith DI, Wong YF: Dysregulated micrornas and their predicted targets associated with endometrioid endometrial adenocarcinoma in hong kong women. Int J Cancer 2009;124:1358-1365.

Chien J, Staub J, Avula R, Zhang H, Liu W, Hartmann LC, Kaufmann SH, Smith DI, Shridhar V: Epigenetic silencing of tceal7 (bex4) in ovarian cancer. Oncogene 2005;24:5089-5100.

Huang CY, Chen YM, Zhao JJ, Chen YB, Jiang SS, Yan SM, Zhao BW, Pan K, Wang DD, Lv L, Li YF, Wang W, Zhou ZW, Xia JC: Decreased expression of transcription elongation factor a-like 7 is associated with gastric adenocarcinoma prognosis. PLoS One 2013;8:e54671.

Chien J, Narita K, Rattan R, Giri S, Shridhar R, Staub J, Beleford D, Lai J, Roberts LR, Molina J, Kaufmann SH, Prendergast GC, Shridhar V: A role for candidate tumor-suppressor gene tceal7 in the regulation of c-myc activity, cyclin d1 levels and cellular transformation. Oncogene 2008;27:7223-7234.

Lafferty-Whyte K, Bilsland A, Hoare SF, Burns S, Zaffaroni N, Cairney CJ, Keith WN: Tceal7 inhibition of c-myc activity in alternative lengthening of telomeres regulates htert expression. Neoplasia 2010;12:405414.

Rattan R, Narita K, Chien J, Maguire JL, Shridhar R, Giri S, Shridhar V: Tceal7, a putative tumor suppressor gene, negatively regulates nf-kappab pathway. Oncogene 2010;29:1362-1373.

Mercader A, Valbuena D, Simon C: Human embryo culture. Methods Enzymol 2006;420:3-18.

Livak KJ, Schmittgen TD: Analysis of relative gene expression data using real-time quantitative pcr and the 2(-delta delta c(t)) method. Methods 2001;25:402-408.

Boren T, Xiong Y, Hakam A, Wenham R, Apte S, Wei Z, Kamath S, Chen DT, Dressman H, Lancaster JM: Micrornas and their target messenger rnas associated with endometrial carcinogenesis. Gynecol Oncol 2008;110:206-215.

2 Zhang L, Volinia S, Bonome T, Calin GA, Greshock J, Yang N, Liu CG, Giannakakis A, Alexiou P, Hasegawa K, Johnstone CN, Megraw MS, Adams S, Lassus H, Huang J, Kaur S, Liang S, Sethupathy P, Leminen A, Simossis VA, Sandaltzopoulos R, Naomoto Y, Katsaros D, Gimotty PA, DeMichele A, Huang Q, Butzow R, Rustgi AK, Weber BL, Birrer MJ, Hatzigeorgiou AG, Croce CM, Coukos G: Genomic and epigenetic alterations deregulate microrna expression in human epithelial ovarian cancer. Proc Natl Acad Sci U S A 2008;105:7004-7009. Wyman SK, Parkin RK, Mitchell PS, Fritz BR, O'Briant K, Godwin AK, Urban N, Drescher CW, Knudsen BS, Tewari M: Repertoire of micrornas in epithelial ovarian cancer as determined by next generation sequencing of small rna cdna libraries. PLoS One 2009;4:e5311.

Martinez I, Gardiner AS, Board KF, Monzon FA, Edwards RP, Khan SA: Human papillomavirus type 16 reduces the expression of microrna-218 in cervical carcinoma cells. Oncogene 2008;27:2575-2582. Tang T, Wong HK, Gu W, Yu MY, To KF, Wang CC, Wong YF, Cheung TH, Chung TK, Choy KW: Microrna-182 plays an onco-mirna role in cervical cancer. Gynecol Oncol 2013;129:199-208.

Gupta A, Caffrey E, Callagy G, Gupta S: Oestrogen-dependent regulation of mirna biogenesis: Many ways to skin the cat. Biochem Soc Trans 2012;40:752-758. 
27 Yan D, Dong XD, Chen X, Yao S, Wang L, Wang J, Wang C, Hu DN, Qu J, Tu L: Role of microrna-182 in posterior uveal melanoma: Regulation of tumor development through mitf, bcl2 and cyclin d2. PLoS One 2012; 7:e40967.

28 Sun Y, Fang R, Li C, Li L, Li F, Ye X, Chen H: Hsa-mir-182 suppresses lung tumorigenesis through down regulation of rgs17 expression in vitro. Biochem Biophys Res Commun 2010;396:501-507.

29 Kong WQ Bai R, Liu T, Cai CL, Liu M, Li X, Tang H: Microrna-182 targets camp-responsive element-binding protein 1 and suppresses cell growth in human gastric adenocarcinoma. FEBS J 2012;279:1252-1260.

-30 Guttilla IK, White BA: Coordinate regulation of foxo1 by mir-27a, mir-96, and mir-182 in breast cancer cells. J Biol Chem 2009;284:23204-23216.

-31 Segura MF, Hanniford D, Menendez S, Reavie L, Zou X, Alvarez-Diaz S, Zakrzewski J, Blochin E, Rose A, Bogunovic D, Polsky D, Wei J, Lee P, Belitskaya-Levy I, Bhardwaj N, Osman I, Hernando E: Aberrant mir182 expression promotes melanoma metastasis by repressing foxo3 and microphthalmia-associated transcription factor. Proc Natl Acad Sci U S A 2009;106:1814-1819.

32 Wang YQ Guo RD, Guo RM, Sheng W, Yin LR: Microrna-182 promotes cell growth, invasion, and chemoresistance by targeting programmed cell death 4 (pdcd4) in human ovarian carcinomas. J Cell Biochem 2013;114:1464-1473.

33 Liu H, Du L, Wen Z, Yang Y, Li J, Wang L, Zhang X, Liu Y, Dong Z, Li W, Zheng G, Wang C: Up-regulation of mir182 expression in colorectal cancer tissues and its prognostic value. Int J Colorectal Dis 2013;28:697-703.

34 Wang J, Li J, Shen J, Wang C, Yang L, Zhang X: Microrna-182 downregulates metastasis suppressor 1 and contributes to metastasis of hepatocellular carcinoma. BMC Cancer 2012;12:227.

-35 Liu Z, Liu J, Segura MF, Shao C, Lee P, Gong Y, Hernando E, Wei JJ: Mir-182 overexpression in tumourigenesis of high-grade serous ovarian carcinoma. J Pathol 2012;228:204-215.

-36 Schaefer A, Jung M, Mollenkopf HJ, Wagner I, Stephan C, Jentzmik F, Miller K, Lein M, Kristiansen G, Jung K: Diagnostic and prognostic implications of microrna profiling in prostate carcinoma. Int J Cancer 2010;126:1166-1176. 